\title{
Clinical Risk Factors Influencing Dental Developmental Disturbances in Childhood Cancer Survivors
}

\author{
Chung-Min Kang, DDS, PhD ${ }^{1,2}$ \\ Seung Min Hahn, MD3,4 \\ Hyo Sun Kim, MD,3,4 \\ Chuhl Joo Lyu, MD, PhD 3,4 \\ Jae-Ho Lee, DDS, PhD ${ }^{1}$ \\ Jinae Lee, $\mathrm{PhD}^{5}$ \\ Jung Woo Han, MD ${ }^{3,4}$
}

\begin{abstract}
Purpose
Although studies regarding dental developmental disturbances after childhood cancer treatment have increased, they have many limitations. Studies analyzing the significance of independent clinical risk factors with regard to the dental health status are also rare. We aimed to investigate the risk factors for dental developmental disturbances, particularly severe disturbances, in childhood cancer survivors (CCS).
\end{abstract}

\section{Materials and Methods}

Oral examinations and retrospective reviews of medical and panoramic radiographs were performed for 196 CCS (mean age, 15.6 years). Cancer type, age at diagnosis, treatment modality, type and accumulated dose of administered drugs, and dose and site of radiation were recorded. Dental developmental disturbances were diagnosed using panoramic radiographs and graded for severity according to the Modified Dental Defect Index (MDDI). Descriptive statistics and multivariate analyses were performed to determine the association between dental abnormalities and clinical factors.

\section{Results}

In total, 109 CCS (55.6\%) exhibited at least one dental anomaly, and the median value of MDDI was 2.5. Microdontia (30.6\%) was the most prevalent anomaly, followed by tooth agenesis (20.4\%), V-shaped roots (14.8\%), and taurodontism (10.2\%). Multivariate analysis revealed that a young age at diagnosis ( $\leq 3$ years), a history of hematopoietic stem cell transplantation, the use of multiple classes of chemotherapeutic agents ( $\geq 4$ classes), and the use of heavy metal agents were significant risk factors for severe dental disturbances.

\section{Conclusion}

CCS with any of the above risk factors for severe developmental disturbances should be comprehensively followed up to minimize adverse consequences to their dental development and preserve their future dental health.
Correspondence: Jung Woo Han, MD College of Medicine, 50 Yonsei-ro, Seodaemun-gu, Seoul 03722, Korea

Tel: 82-2-2228-2050

Fax: 82-2-393-9118

E-mail:JWHAN@yuhs.ac

\section{Key words}

Neoplasms, Survivors, Adverse effects, Odontogenesis, Tooth abnormalities

\section{Introduction}

The survival of patients with childhood cancer has increased because of current multimodal therapies, and research has been increasingly focusing on the future quality of life of these survivors. The long-term dental effects of cancer therapy in children include several irreversible developmental anomalies, such as tooth agenesis, microdontia, enamel hypoplasia, disturbed root development, and taurodontism [1]. More than $90 \%$ of childhood cancer survivors (CCS) exhibit an oral complication of some type [2].

Both chemotherapeutic agents and radiation therapy, which should ideally eradicate only tumor cells and cause minimal damage to normal tissue, lack specificity, and this results in the occurrence of dental and facial developmental anomalies. The severity of effects on dentofacial structures was found to be associated with the stage of odontogenesis, 
age at cancer diagnosis, type of treatment received, and dose and field of irradiation [3]. Chemotherapy and radiation therapy in children have negative effects on dental development, which is a unique process that occurs during the first 15-16 years of life. Existing reports have found that patients treated with chemotherapy at less than 5 years of age may be at a greater risk for dental anomalies because of the proliferation of dental stem cells during this period [4].

Although there are multiple studies investigating dental developmental disturbances after childhood cancer treatment, they have many limitations, including a small number of participants, the use of questionnaires instead of clinical examinations, and the use of registry databases $[5,6]$. Studies analyzing the significance of independent clinical risk factors with regard to dental health status are also rare. In the present study, we used comprehensive clinical examinations and panoramic radiographs to evaluate the severity of dental developmental disturbances in CCS in an established longterm follow-up clinic using a newly developed scoring system.

\section{Materials and Methods}

\section{Study population}

In total, 196 CCS who visited the long-term follow-up clinic in the childhood cancer center at Yonsei Cancer Center, Yonsei University Health System between December 13, 2014 and October 1, 2016 were included in this study. The mini- mum follow-up duration was 2 years after the completion of childhood cancer therapy. The primary outcome was the occurrence of dental complications at least 2 years after the completion of cancer therapy.

\section{Demographics and evaluated risk factors}

Oral examinations were performed in conjunction with a retrospective review of medical and panoramic radiography data; the cancer type, age at cancer diagnosis, treatment modality, type and accumulated dose of administered drugs, and dose and site of radiation were recorded. The patients were divided into three groups according to the age at cancer diagnosis: youngest ( $\leq 3$ years old), middle ( $3-5$ years), and oldest ( $\geq 5$ years). The class of chemotherapeutic agents was defined by the mechanisms of action for each agent. The total number of chemotherapeutic agent classes used for each survivor was recorded to estimate the toxicity of chemotherapy; "multiple classes" was defined as $\geq 4$ classes. The radiation dose was defined as the maximal dose of radiation delivered to specific body regions, including the head and neck.

\section{Diagnosis of dental developmental disturbances}

Dental developmental disturbances were diagnosed using dental examinations, panoramic radiography, and dental histories. Clinical and radiographic data were reviewed by pediatric dental specialists. Tooth agenesis was defined as the congenital absence of a permanent tooth or tooth germ (third molars were excluded). Microdontia was defined as a tooth size that was smaller than normal (third molars and the maxillary lateral incisor, which is commonly affected, were

Table 1. Classification of dental developmental disturbances with the Modified Dental Defect Index (MDDI)

\begin{tabular}{|c|c|c|}
\hline Site & Classification & MDDI \\
\hline \multirow[t]{3}{*}{ Crown } & Normal crown development & 0 \\
\hline & Mild enamel hypoplasia diagnosed as developmental enamel defect & 1 \\
\hline & Severe enamel hypoplasia diagnosed as notches on proximal surfaces & 2 \\
\hline \multirow[t]{5}{*}{ Root } & Normal root development, $\mathrm{R} / \mathrm{C}$ ratio $>1.6$ & 0 \\
\hline & Taurodontism (taurodontic index $>20$ ) & 1 \\
\hline & $\begin{array}{l}\text { Arrested root development with shortened tapered } V \text {-shaped roots } \\
(\mathrm{R} / \mathrm{C} \text { ratio=0.8-1.6; greater than } 50 \% \text { normal root length) }\end{array}$ & 1 \\
\hline & $\begin{array}{l}\text { Arrested root development with shortened blunted (U-shaped) roots } \\
(\mathrm{R} / \mathrm{C} \text { ratio }<0.8 \text {; less than } 50 \% \text { normal root length) }\end{array}$ & 3 \\
\hline & Total arrested root development & 5 \\
\hline \multirow[t]{2}{*}{ Crown and root } & Microdontia (mesiodistal crown size was about half or less than the expected tooth size) & 4 \\
\hline & Tooth agenesis & 6 \\
\hline
\end{tabular}

In multi-rooted teeth, the longest root was used in the calculation. $\mathrm{R} / \mathrm{C}$ ratio, relative ratio between root $(\mathrm{R})$ and crown $(\mathrm{C})$ length. 


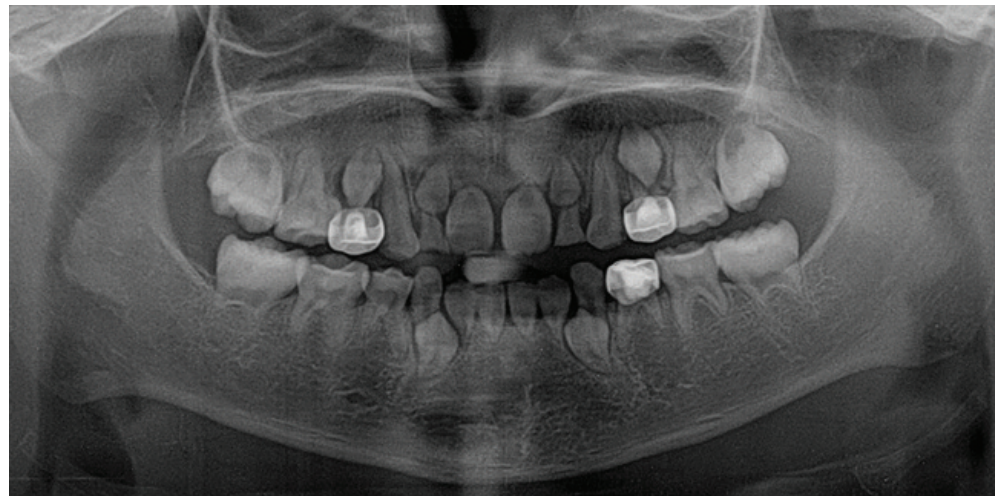

B

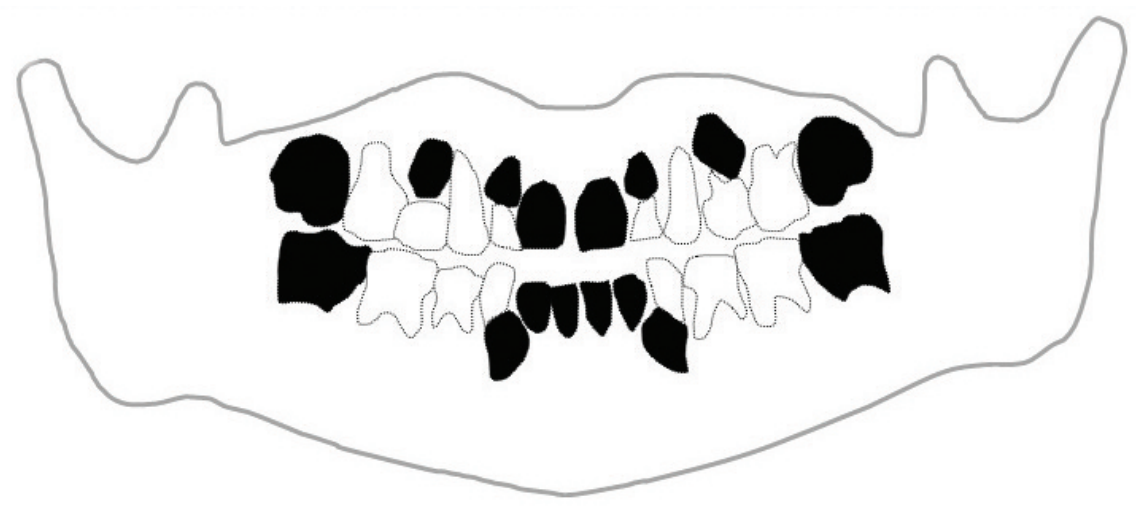

\begin{tabular}{|c|c|c|c|c|c|c|c|c|c|c|c|c|c|c|c|c|}
\hline & Upp & er riç & & & & & & & & & & & & \multicolumn{3}{|c|}{ Upper left } \\
\hline Tooth number & 18 & 17 & 16 & 15 & 14 & 13 & 12 & 11 & 21 & 22 & 23 & 24 & 25 & 26 & 27 & 28 \\
\hline MDDI & - & 6 & 5 & 6 & - & 6 & 4 & 5 & 5 & 4 & 6 & - & 6 & 5 & 6 & - \\
\hline MDDI & - & 6 & 5 & 6 & 6 & - & 5 & 5 & 5 & 5 & - & 6 & 6 & 5 & 6 & - \\
\hline Tooth number & 48 & 47 & 46 & 45 & 44 & 43 & 42 & 41 & 31 & 32 & 33 & 34 & 35 & & 37 & 38 \\
\hline
\end{tabular}

Fig. 1. A panoramic radiograph (A) and schematic presentation (B) of the method of calculation of the Modified Dental Defect Index (MDDI) score for a patient (7 years and 11 months, male) with Ewing's sarcoma. The patient received 45 Gy of radiation to the ethmoid region at 3 years and 2 months of age and chemotherapy with doxorubicin $\left(300 \mathrm{mg} / \mathrm{m}^{2}\right)$ at 1 year and 7 months of age. Twelve permanent teeth are missing, and the development of all present teeth is severely affected. The total MDDI score is 130 .

excluded). The criteria for classifying data as undetermined were as follows: ongoing tooth development with an unclear final morphology, absence of teeth that are not categorized in the agenesis group at a young age, and generally unreliable appearance on panoramic view. With regard to tooth agenesis, a tooth was not considered missing before a certain age as follows: first premolar, $<5$ years; second premolar, $<6$ years; and second molar, $<7$ years.

\section{Modified Dental Defect Index}

We developed the Modified Dental Defect Index (MDDI) to describe the severity of damage to the dentition in the present study (Table 1). This index system is a modified version of the defect index and the dental disturbance classification and severity rating scale proposed by Sonis et al. [4] and Holtta et al. [7]. The MDDI combines abnormalities in 
Table 2. Demographics of the study population

\begin{tabular}{|c|c|c|c|c|c|}
\hline Characteristic & All & $\begin{array}{c}\text { Youngest } \\
\text { ( } \leq 3 \mathrm{yr} \text { ) }\end{array}$ & $\begin{array}{l}\text { Middle } \\
(3-5 \mathrm{yr})\end{array}$ & $\begin{array}{l}\text { Oldest } \\
\text { ( } \geq 5 \text { yr) }\end{array}$ & p-value \\
\hline Sex (male:female) & $127: 69$ & $44: 20$ & $30: 15$ & $53: 34$ & \\
\hline Age at diagnosis (yr) & $4.7(0-16.4)$ & $1.6(0-3.0)$ & $4.0(3.0-4.9)$ & $8.6(5.0-16.4)$ & $<0.001$ \\
\hline Age at completion (yr) & $7.1(0.4-16.9)$ & $3.0(0.4-11.0)$ & $6.3(3.9-13.2)$ & $10.0(5.9-16.9)$ & $<0.001$ \\
\hline Treatment term & $1.1(0-10.25)$ & $0.9(0-10.25)$ & $3.0(0.3-8.5)$ & $1.0(0-6.8)$ & 0.003 \\
\hline Evaluation age & $14.9(4.6-33.9)$ & $12.7(4.6-25.2)$ & $13.1(6.4-29.4)$ & $17.4(9.4-33.9)$ & $<0.001$ \\
\hline Follow-up term & $6.9(2.1-22.5)$ & $9.5(2.3-21.8)$ & $7.3(2.1-22.5)$ & $6.1(2.1-22.0)$ & 0.001 \\
\hline \multicolumn{6}{|l|}{ Diagnosis } \\
\hline ALL & $71(36.2)$ & $15(23.4)$ & $28(63.6)$ & $28(31.8)$ & $<0.001$ \\
\hline AML & $7(3.6)$ & $2(3.1)$ & 0 & $5(5.7)$ & 0.246 \\
\hline Lymphoma & $23(11.7)$ & $6(9.4)$ & $4(9.1)$ & $13(14.8)$ & 0.490 \\
\hline Brain tumor & $22(11.2)$ & $3(4.7)$ & $1(2.3)$ & $18(20.5)$ & 0.001 \\
\hline Sarcoma & $18(9.2)$ & $4(6.3)$ & $3(6.8)$ & $11(12.5)$ & 0.347 \\
\hline Abdomen & $37(18.9)$ & $23(35.9)$ & $6(13.6)$ & $8(9.1)$ & $<0.001$ \\
\hline Others & $18(9.2)$ & $11(17.2)$ & $2(4.5)$ & $5(5.7)$ & 0.025 \\
\hline
\end{tabular}

Values are presented as median (range) or number (\%). Others indicate Langerhans cell histiocytosis, aplastic anemia, hemophagocytic lymphohistiocytosis, hemophagocytic syndrome. ALL, acute lymphoblastic leukemia; AML, acute myelogenous leukemia.

root length, crown calcification, microdontia, and tooth agenesis, thus representing the overall damage to the permanent dentition with a single index figure. The MDDI was finally calculated by adding the score for each permanent tooth as shown in Fig 1 . The disturbances in each patient were categorized as normal (MDDI, 0$)$, moderately abnormal $(1 \leq$ MDDI < 14), and severely abnormal (MDDI $\geq 14$ ). The cutoff (MDDI, 14) score was set to the median value for the survivors with an abnormal MDDI score $(\geq 1)$.

\section{Statistical analysis}

Dental developmental disturbances and clinical risk factors were evaluated using exact chi-square tests, and KruskalWallis tests. Logistic regression models were used to assess the association between the risk factors and severely abnormal disturbances. Factors that were significant in the univariate analysis and clinically meaningful variables were selected for the multivariate analysis. All statistical analyses were performed using IBM SPSS ver. 23.0 (IBM SPSS Statistics, IBM Corp., Armonk, NY) and OriginPro 2016 software (OriginLab, Northampton, MA).

\section{Ethical statement}

All procedures performed in studies involving human participants were in accordance with the ethical standards of the Institutional Review Board of Yonsei University Dental Hos- pital (approval number: 2-2015-0010). The informed consent was waived.

\section{Results}

\section{Patient characteristics}

Among the 196 survivors, 127 were boys and 69 were girls. The median age at cancer diagnosis and treatment completion was 4.7 and 7.1 years, respectively. The median time since the completion of cancer therapy was 6.9 years (2.122.5). The most prevalent type of cancer was acute lymphoblastic leukemia (36.2\%) (Table 2). The proportion of patients who received chemotherapy was $99.5 \%$, and the median number of agent classes was seven. There was a significant difference in the type of drug treatment received between the three age groups, which included enzymes, alkylating agents, antimetabolites, corticosteroids, and anthracyclines (S1 Table). Radiotherapy was administered to 67 patients (34.2\%), with the dose ranging from 6.0 to 70.2 Gy. Radiotherapy use was most prevalent in the oldest group $(47.9 \%)$, followed by the middle $(27.3 \%)$ and youngest $(17.2 \%)$ groups. Forty-seven patients $(24.0 \%)$ underwent hematopoietic stem cell transplantation (HSCT); the median age at HSCT was 6.1 (0.6-14.8) years (S2 Table). 
Table 3. Prevalence of dental developmental disturbances in childhood cancer survivors (overall cohort and different age groups)

\begin{tabular}{|c|c|c|c|c|c|}
\hline Characteristic & All & $\begin{array}{l}\text { Youngest } \\
(\leq 3 \mathrm{yr})\end{array}$ & $\begin{array}{l}\text { Middle } \\
(3-5 \mathrm{yr})\end{array}$ & $\begin{array}{l}\text { Oldest } \\
(\geq 5 \mathrm{yr})\end{array}$ & p-value \\
\hline Normal & $87(44.4)$ & $13(20.3)$ & $19(43.2)$ & $55(62.5)$ & $<0.001$ \\
\hline Dental disturbances & $109(55.6)$ & $51(79.7)$ & $25(56.8)$ & $33(37.5)$ & $<0.001$ \\
\hline Tooth agenesis & $40(20.4)$ & $25(39.1)$ & $7(15.9)$ & $8(9.1)$ & $<0.001$ \\
\hline Microdontia & $60(30.6)$ & $37(57.8)$ & $13(29.5)$ & $10(11.4)$ & $<0.001$ \\
\hline Mild enamel hypoplasia & $10(5.1)$ & $4(6.3)$ & $2(4.5)$ & $4(4.5)$ & 0.879 \\
\hline Severe enamel hypoplasia & $14(7.1)$ & $11(17.2)$ & $3(6.8)$ & 0 & $<0.001$ \\
\hline V-shaped root & $29(14.8)$ & $13(20.3)$ & $8(18.2)$ & $8(9.1)$ & 0.121 \\
\hline U-shaped root & $19(9.7)$ & $4(6.3)$ & $5(11.4)$ & $10(11.4)$ & 0.525 \\
\hline Total arrested root development & $1(0.5)$ & $1(1.6)$ & 0 & 0 & 0.355 \\
\hline Taurodontism & $20(10.2)$ & $7(10.9)$ & $8(18.2)$ & $5(5.7)$ & 0.080 \\
\hline Total MDDI score & $2.5(0-130)$ & $18(0-130)$ & $6(0-44)$ & $0(0-36)$ & $<0.001$ \\
\hline
\end{tabular}

Values are presented as number (\%) and median (range). MDDI, Modified Dental Defect Index.

A

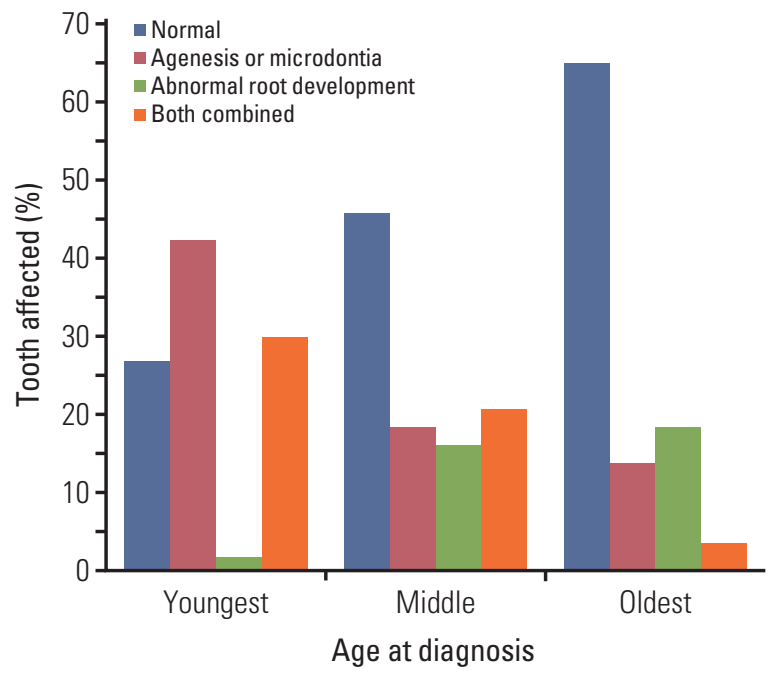

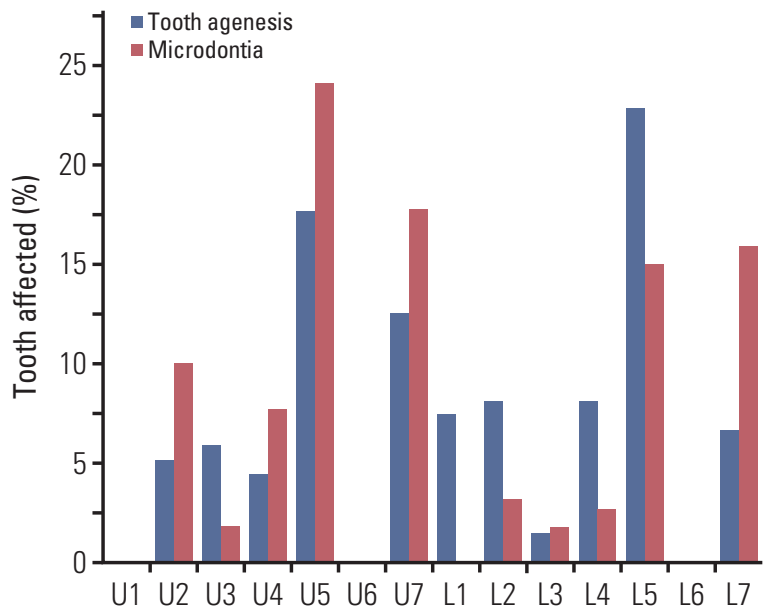

Fig. 2. (A) Proportions of patients with tooth agenesis, microdontia, abnormal root development, and combined anomalies in the three age groups of childhood cancer survivors (youngest, $\leq 3$ years; middle, $3-5$ years; oldest, $\geq 5$ years). The prevalence of agenesis or microdontia is the highest in the youngest group, while that of abnormal root development exhibits a tendency to increase in the older groups. (B) Distribution of teeth affected by tooth agenesis and microdontia. The data show that microdontia is most common in the maxillary second premolars and second molars, while tooth agenesis is most common in the maxillary and mandibular second premolars. U1, upper (maxillary) central incisor.

\section{Prevalence of dental developmental disturbances}

The panoramic radiographs of 87 patients $(44.4 \%)$ showed no dental abnormalities at the most recent examination; those of the remaining 109 patients $(55.6 \%)$ showed at least one abnormality. Microdontia $(30.6 \%)$ was the most prevalent, followed by tooth agenesis (20.4\%), V-shaped roots (14.8\%), and taurodontism $(10.2 \%)$ (Table 3$)$. In the youngest group, $42.2 \%$ of patients exhibited tooth agenesis or microdontia. Abnormal root development was less frequently observed in 

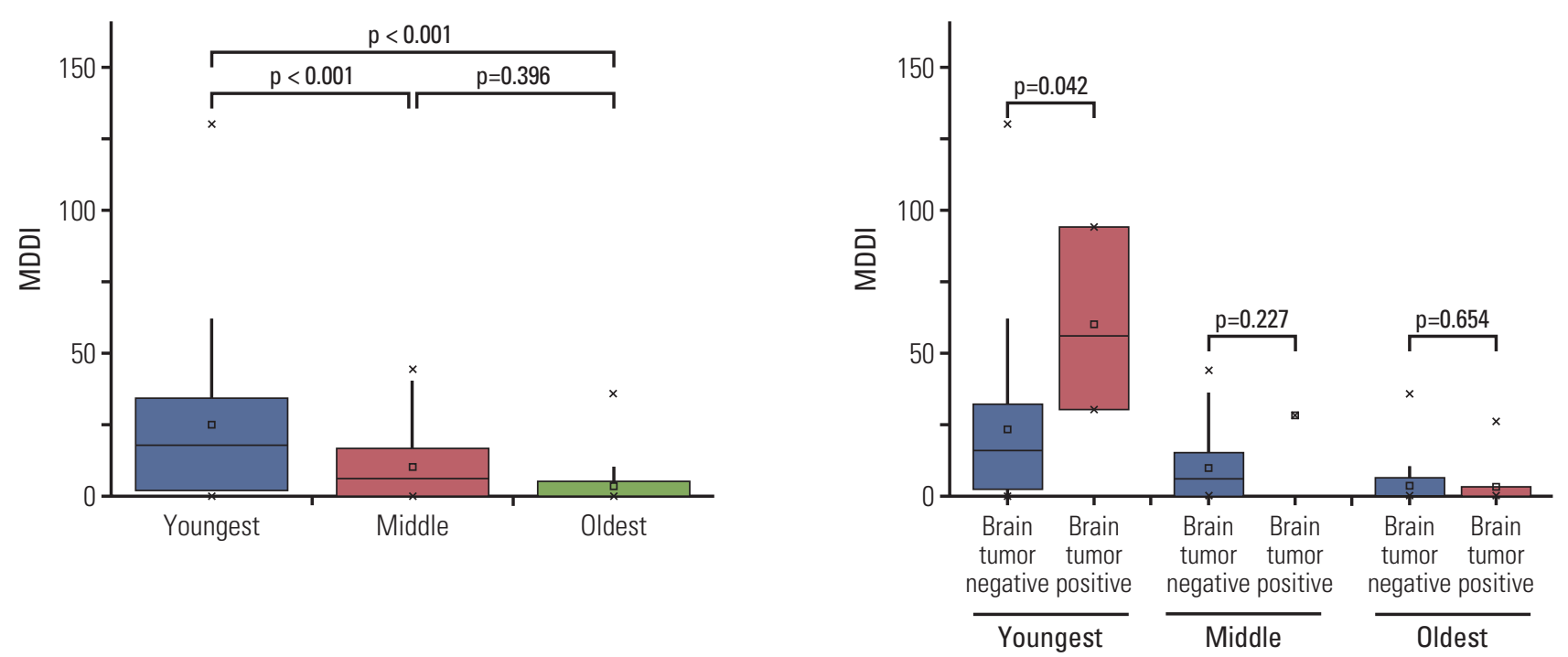

C
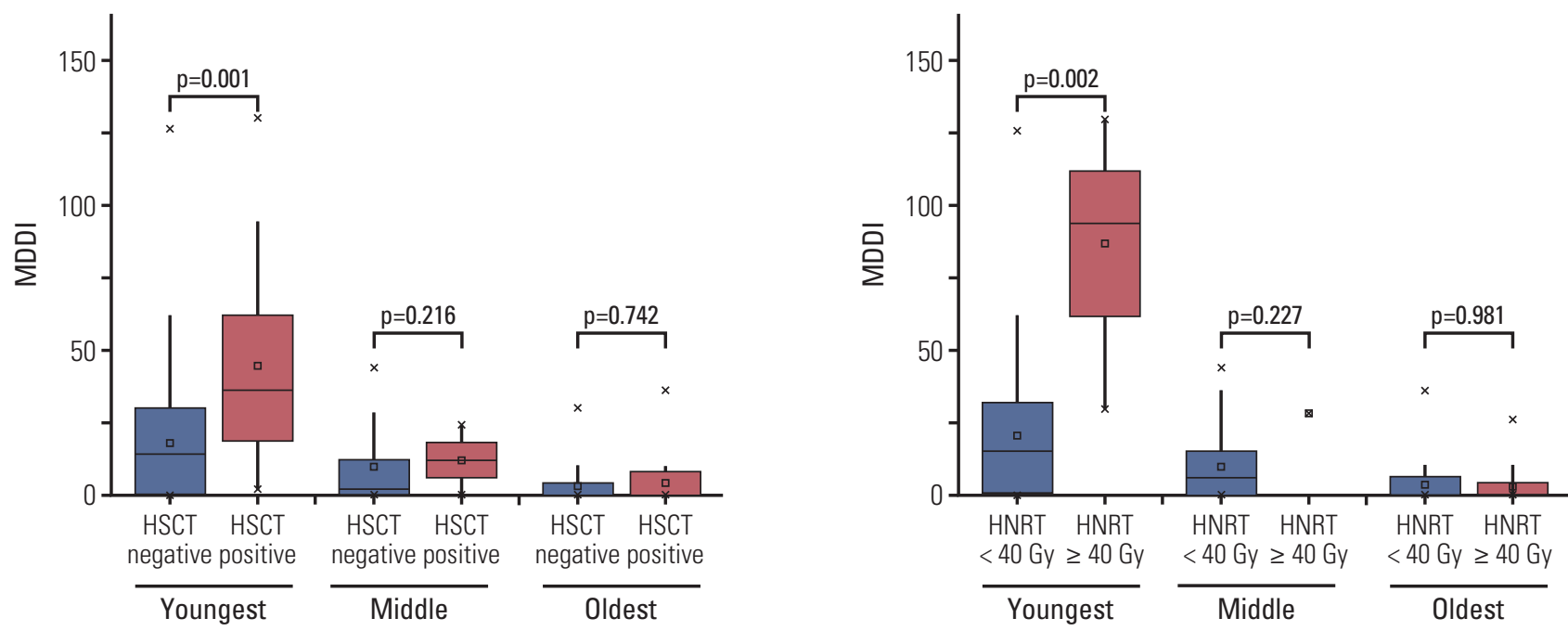

Fig. 3. Relationship between Modified Dental Defect Index (MDDI) scores and treatment factors in the three age groups of childhood cancer survivors (youngest, $\leq 3$ years; middle, $3-5$ years; oldest, $\geq 5$ years). (A) The MDDI score for the youngest group is significantly different compared to the middle and oldest groups $(\mathrm{p}<0.001)$. (B) The MDDI score is significantly higher in the youngest group diagnosed with brain tumors $(\mathrm{p}=0.042)$. (C) The MDDI score is significantly higher in youngest group treated with hematopoietic stem cell transplantation ( $\mathrm{p}=0.001$ ). (D) The MDDI score is significantly higher in youngest group treated with head and neck radiation therapy at a dose of $\geq 40 \mathrm{~Gy}$ ( $\mathrm{p}=0.002)$. (Continued to the next page)

this group compared with the other groups (1.6\%) (Fig. 2A). In the oldest group, abnormal root development $(18.2 \%)$ was more prevalent than agenesis or microdontia $(13.6 \%)$, while the prevalence of combined disturbances $(3.4 \%)$ in the youngest group was markedly lower than that in the other groups. The most common teeth exhibiting agenesis were the mandibular $(22.8 \%)$ and maxillary (17.7\%) second premolars. Microdontia was the most prevalent in the maxillary second premolar $(24.1 \%)$, followed by the maxillary second molar (17.7\%) (Fig. 2B). 
$\mathbf{E}$
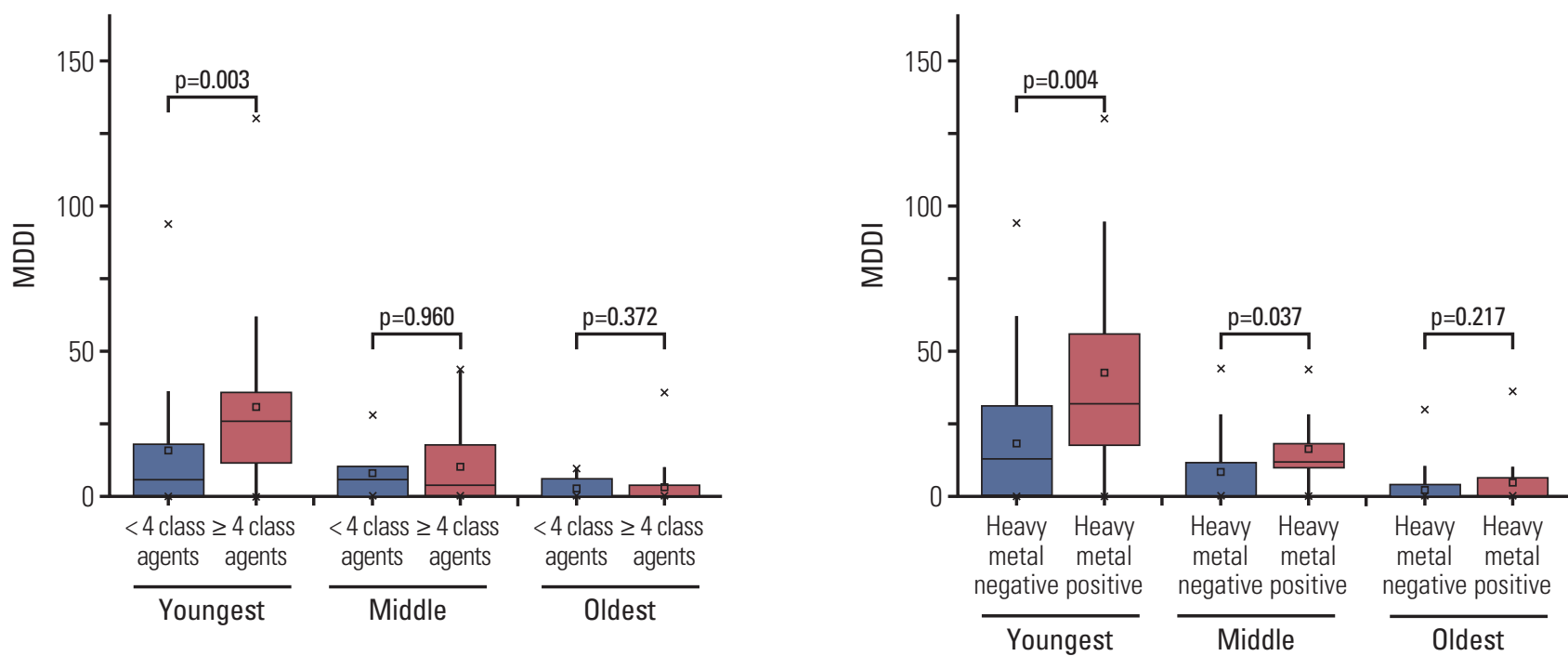

Fig. 3. (Continued from the previous page) (E) The MDDI score is significantly higher in the youngest group receiving $\geq 4$ classes of chemotherapeutic agents $(\mathrm{p}=0.003)$. (F) The use of heavy metal agents significantly increases the MDDI score in the youngest and middle groups ( $\mathrm{p}=0.004$ and $\mathrm{p}=0.037$, respectively).

Table 4. Multivariate analysis of potential risk factors for severely abnormal Modified Dental Defect Index (MDDI) compared to normal MDDI group

\begin{tabular}{|c|c|c|c|}
\hline Characteristic & Odds ratio & 95\% Confidence interval & p-value \\
\hline \multicolumn{4}{|l|}{ Sex } \\
\hline Male vs. female & 0.753 & $0.255-2.217$ & 0.606 \\
\hline \multicolumn{4}{|l|}{ Age at diagnosis } \\
\hline Youngest ( $\leq 3 \mathrm{yr})$ & 265.757 & $37.037-1,906.915$ & $<0.001$ \\
\hline Middle ( $>3 \mathrm{yr}$ and $<5 \mathrm{yr}$ ) & 33.897 & $5.583-205.796$ & $<0.001$ \\
\hline Oldest ( $\geq 5$ yr) & Reference & & \\
\hline \multicolumn{4}{|l|}{ Brain tumor } \\
\hline No vs. brain tumor & 0.420 & $0.016-11.270$ & 0.605 \\
\hline \multicolumn{4}{|l|}{ HNRT dose } \\
\hline$<40$ Gy vs. $\geq 40$ Gy & 2.442 & $0.122-48.983$ & 0.560 \\
\hline \multicolumn{4}{|l|}{ HSCT } \\
\hline Non-HSCT vs. HSCT & 3.875 & $1.016-14.785$ & 0.047 \\
\hline \multicolumn{4}{|c|}{ Multiple class of chemotherapeutic agents } \\
\hline$<4$ class vs. $\geq 4$ class & 8.878 & $2.263-34.822$ & 0.002 \\
\hline \multicolumn{4}{|l|}{ Use of heavy metal agents } \\
\hline No vs. yes & 9.350 & $1.970-44.370$ & 0.005 \\
\hline
\end{tabular}

HNRT, head and neck radiation therapy; HSCT, hematopoietic stem cell transplantation. 


\section{Clinical risk factors for dental developmental distur- bances}

The MDDI score was significantly higher in the youngest group than the other two groups $(p<0.001)$. There was no significant difference in the MDDI score between the other two groups ( $p=0.396)$ (Fig. 3A). The presence of a brain tumor and a history of HSCT were associated with an increased risk for developmental anomalies, particularly in the youngest group ( $p=0.042$ and $p=0.001$, respectively) (Fig. 3B and C). In addition, the MDDI score showed a significant increase with the maximum head and neck radiation therapy (HNRT) dose ( $\geq 40 \mathrm{~Gy}$ and $<40 \mathrm{~Gy}$ ) in the youngest group $(\mathrm{p}=0.002)$ (Fig. 3D). The use of $\geq 4$ classes of chemotherapeutic agents $(p=0.003)$ and the use of heavy metal agents $(p=0.004)$ also demonstrated a significant association with an increased MDDI score in the youngest group (Fig. 3E and F). The linear-by-linear association test was used to examine the linear association between clinical risk factors and MDDI severity outcomes. The use of heavy metal agents significantly affected moderate and severe dental defects (S3 Table). Comparing MDDI according to the age group and clinical risk factors, the score increased in patients with a history of HSCT, heavy metal use, and tumor of the abdomen. Additional risk factors were influenced by the youngest group, which included a history of $\geq 40$ Gy HNRT, the use of alkylating agents, and a diagnosis of brain tumor. A history of sarcoma was a significant risk factor for dental defects in the middle group (S4 Table).

\section{Multivariate analysis of risk factors for severe dental developmental disturbances}

Multivariate analysis revealed that a young age at diagnosis ( $\leq 3$ years), a history of HSCT, the use of $\geq 4$ classes of chemotherapeutic agents, and the use of heavy metal agents were significant risk factors for severely abnormal MDDI scores (Table 4).

\section{Discussion}

We investigated the significant risk factors for dental developmental disturbances in CCS among different age groups and found that childhood cancer treatment often results in developmental disturbances of permanent dentition, with $55.6 \%$ of patients exhibiting at least one dental disturbance. Depending on the age at cancer diagnosis and the intensity of treatment, the dental abnormalities ranged from tooth agenesis $(20.4 \%)$ to microdontia $(30.6 \%)$ and shortened roots caused by the premature arrest of root formation.

In the present study, a young age at cancer diagnosis $(\leq 3$ years) was found to be the most important risk factor for dental developmental anomalies. This finding suggests that tooth development may be affected at any point prior to complete maturation. Our age group classification was based on the standard schedule for tooth development. Calcification of the first premolars begins between 1.5 and 2 years of age, while that of the second premolars and second molars begins between 2 and 2.5 years and 2-2.5 and 3 years, respectively. While other permanent teeth start hard tissue formation within 1 year of birth, premolars and the second molars begin initial calcification 2 years after birth. Therefore, these teeth are vulnerable to environmental factors such as cancer treatment during their development. If teeth do not develop at or near these times, they will grow abnormally, resulting in tooth agenesis or microdontia. Between 3 and 5 years of age, root development begins in teeth that develop early and crown development is ongoing in teeth that develop late. Cancer treatment administered during this period is believed to cause disturbances to both crowns and roots. The present study clearly demonstrates that the prevalence of tooth agenesis and microdontia decreases and that of abnormal root development tends to increase with age. Previous studies have shown that children treated before and approximately 5 years of age were at a high risk for dental disturbances due to localized or systemic insults on ameloblasts and odontoblasts, which arrest dental growth $[2,3]$. Since the dental development process is very active between 3 and 5 years of age, the high frequency of treatment-related dental abnormalities found in our study is not surprising. In contrast, studies have suggested that the prevalence of dental developmental disturbances does not change with the age at cancer diagnosis $[8,9]$. These differences in findings are probably caused by differences in the treatment protocol and the inclusion of small groups confined to a single diagnosis. Additionally, variations in the timing of tooth formation could also be a possible reason.

Chemotherapeutic drugs can affect the development of permanent teeth. The severity of tooth-related abnormalities secondary to cancer treatment is related to the age at the time of treatment, drug dosage, and treatment duration [10]. Disturbed odontogenesis has been reported after the administration of several chemotherapeutic agents, including anthracyclines, cyclophosphamide, vincristine, and actinomycin D [11]. Interestingly, heavy metal agents such as cisplatin and carboplatin also alter normal dental development. These drugs are meant to interfere with replication and the mitotic processes of cancer cells only, given the significant side effects associated with healthy cell destruction [12]. In a previous study, exposure to cisplatin elicited a minor decrease in precursor cells in the epithelial sheath and dental 
pulp stem cells, although the toxicity was similar to that caused by other agents $[13,14]$.

Radiation therapy is often implicated in development disorders of the body; however, the minimum dose that alters the development of the dentition remains unclear. Dental defects are more severe in children subjected to head and neck radiotherapy and total body irradiation combined with chemotherapy than in children subjected to chemotherapy alone $[8,9]$. In a previous study, nine of 10 patients who received fractionated total body irradiation exhibited severe root defects, whereas none of the patients who received chemotherapy alone developed severe defects [15]. A previous study reported that a radiation dose of 4 Gy would affect dental development [16], while another reported that doses of $10 \mathrm{~Gy}$ and $30 \mathrm{~Gy}$ are enough to cause permanent damage to mature ameloblasts and arrest dental development, respectively [17]. In the present study, we observed a significant increase in the MDDI score in a dose-dependent pattern $(\geq 40 \mathrm{~Gy}$ ) in children younger than 3 years. High-dose HNRT was not found to be a significant factor in the multivariate analysis because most patients who received $\geq 40$ Gy were above 5 years of age; radiotherapy was avoided in the youngest group. However, in the univariate analysis, a head and neck dose of $\geq 40$ Gy influenced the MDDI score for the youngest group, but not the score for the oldest group. Therefore, the age of the patients was more important than HNRT itself.

In the present study, the youngest patients who received HSCT exhibited a statistically higher MDDI score compared to the other groups, suggesting that HSCT may affect the prevalence and severity of dental anomalies. Although relevant studies regarding HSCT recipients are few, our patients exhibited more dental anomalies than those who received conventional chemotherapy. In our study, the most severe disturbances in the root/crown ratio occurred in middleaged patients who received HSCT. The conditioning regimen is considered the primary reason for severe dental disturbances, because it frequently includes high-dose chemotherapy and total body irradiation [18].

The most common dental anomaly in the present study was microdontia $(33.7 \%)$. The prevalence of microdontia ranges from $4 \%$ to $78 \%$ in CCS $[4,8,15]$. This variation is probably due to different ages at cancer diagnosis; younger patients were at a higher risk of microdontia. The prevalence of microdontia in the present study clearly exceeded that in Japanese children (1.9\%), Iranian individuals aged below 20 years (2\%), and Korean individuals (11.2\%) [19-21]. The maxillary premolar was most commonly affected by microdontia, followed by the maxillary second molar. Of note, tooth agenesis and microdontia of the second premolars and second molars occurred most frequently in children exposed before 3 years of age. The most common root malformation in the present study was V-shaped roots (29.8\%). Altered odontoblastic activity, a consequence of abnormal secretory function of microtubules and complex changes in inter- and intracellular relationships, can result in short, thin, and blunt roots. Some chemotherapeutic agents such as vinblastine and vincristine can affect mature secretory odontoblasts and ameloblasts.

This study has a number of strengths. It provided comprehensive clinical evaluations for the dental health status and included a large number of CCS. The novel MDDI system we developed was able to provide specific measurements for the severity of damage to the permanent dentition. However, the measurements of crown size based on clinical examination were not considered as reliable. Dental plaster casts should be used for accurate measurements. In addition, the heterogeneous nature of the clinical course of each case made it difficult to evaluate the differential dental effects according to cancer treatment.

A multidisciplinary approach involving oncologists, pediatric dentists, and other related health professionals is essential in caring for the child before, during and after any cancer therapy. We recommend completion of a baseline dental check as soon as the patient can cooperate in taking a panoramic radiograph. The risk factors identified in this study can help clinicians predict dental developmental disturbances. For patients with a higher risk, it will be necessary to visit the dental clinic at least every six months (or more often if issues such as oral mucositis, xerostomia, or trismus are present) [22]. Periodic surveillance facilitates detection of abnormal tooth development, enabling pediatric dentists to intervene earlier and promote a more aggressive treatment plan. In cases of high dose HNRT to patients under 3 years, the use of lead-lined stents, prostheses, and shields should be discussed with the radiation oncologist $[22,23]$. In addition, informed consent should include a discussion of the dental developmental disturbances that can possibly occur as a result of the proposed cancer treatment.

In conclusion, these results suggest that severe dental developmental disturbances were significantly associated with a cancer diagnosis at $\leq 3$ years of age, a history of HSCT, the use of $\geq 4$ classes of chemotherapeutic agents, and the use of heavy metal agents. This study provides information that can assist clinicians in early detection, prevention, and timely intervention.

\section{Electronic Supplementary Material}

Supplementary materials are available at Cancer Research and Treatment website (http:// www.e-crt.org). 


\section{Conflicts of Interest}

Conflict of interest relevant to this article was not reported.

\section{Acknowledgments}

This work was supported by the long-term follow-up clinic for childhood cancer survivors at Yonsei Cancer Center, Yonsei University Health System, Seoul, Korea.

\section{References}

1. Kaste SC, Hopkins KP, Jones D, Crom D, Greenwald CA, Santana VM. Dental abnormalities in children treated for acute lymphoblastic leukemia. Leukemia. 1997;11:792-6.

2. Avsar A, Elli M, Darka O, Pinarli G. Long-term effects of chemotherapy on caries formation, dental development, and salivary factors in childhood cancer survivors. Oral Surg Oral Med Oral Pathol Oral Radiol Endod. 2007;104:781-9.

3. Carrillo CM, Correa FN, Lopes NN, Fava M, Odone Filho V. Dental anomalies in children submitted to antineoplastic therapy. Clinics (Sao Paulo). 2014;69:433-7.

4. Sonis AL, Tarbell N, Valachovic RW, Gelber R, Schwenn M, Sallan S. Dentofacial development in long-term survivors of acute lymphoblastic leukemia: a comparison of three treatment modalities. Cancer. 1990;66:2645-52.

5. Gawade PL, Hudson MM, Kaste SC, Neglia JP, Constine LS, Robison LL, et al. A systematic review of dental late effects in survivors of childhood cancer. Pediatr Blood Cancer. 2014;61: 407-16.

6. Proc P, Szczepanska J, Skiba A, Zubowska M, Fendler W, Mlynarski W. Dental anomalies as late adverse effect among young children treated for cancer. Cancer Res Treat. 2016;48: 658-67.

7. Holtta P, Alaluusua S, Saarinen-Pihkala UM, Wolf J, Nystrom M, Hovi L. Long-term adverse effects on dentition in children with poor-risk neuroblastoma treated with high-dose chemotherapy and autologous stem cell transplantation with or without total body irradiation. Bone Marrow Transplant. 2002;29: 121-7.

8. Oguz A, Cetiner S, Karadeniz C, Alpaslan G, Alpaslan C, Pinarli G. Long-term effects of chemotherapy on orodental structures in children with non-Hodgkin's lymphoma. Eur J Oral Sci. 2004;112:8-11.

9. Duggal MS. Root surface areas in long-term survivors of childhood cancer. Oral Oncol. 2003;39:178-83.

10. Dahllof G, Rozell B, Forsberg CM, Borgstrom B. Histologic changes in dental morphology induced by high dose chemotherapy and total body irradiation. Oral Surg Oral Med Oral Pathol. 1994;77:56-60.

11. Lyaruu DM, van Duin MA, Bervoets TJ, Bronckers AL, Woltgens JH. Daunorubicin-induced pathology in the developing hamster molar tooth germ in vitro. Cancer Detect Prev.
1999;23:343-50.

12. Weidmann AG, Komor AC, Barton JK. Targeted chemotherapy with metal complexes. Comments Mod Chem A Comments Inorg Chem. 2014;34:114-23.

13. Satoh H, Uesugi $Y$, Kawabata T, Mori K, Fujii F, Kashimoto $Y$, et al. Morphological classification of dental lesions induced by various antitumor drugs in mice. Toxicol Pathol. 2001;29: 292-9.

14. Seifrtova M, Havelek R, Cmielova J, Jiroutova A, Soukup T, Bruckova $\mathrm{L}$, et al. The response of human ectomesenchymal dental pulp stem cells to cisplatin treatment. Int Endod J. 2012;45:401-12.

15. Holtta P, Alaluusua S, Saarinen-Pihkala UM, Peltola J, Hovi L. Agenesis and microdontia of permanent teeth as late adverse effects after stem cell transplantation in young children. Cancer. 2005;103:181-90.

16. Goho C. Chemoradiation therapy: effect on dental development. Pediatr Dent. 1993;15:6-12.

17. Dury DC, Roberts MW, Miser JS, Folio J. Dental root agenesis secondary to irradiation therapy in a case of rhabdomyosarcoma of the middle ear. Oral Surg Oral Med Oral Pathol. 1984;57:595-9.

18. Nasman M, Forsberg CM, Dahllof G. Long-term dental development in children after treatment for malignant disease. Eur J Orthod. 1997;19:151-9.

19. Ooshima T, Ishida R, Mishima K, Sobue S. The prevalence of developmental anomalies of teeth and their association with tooth size in the primary and permanent dentitions of 1650 Japanese children. Int J Paediatr Dent. 1996;6:87-94.

20. Chung CJ, Han JH, Kim KH. The pattern and prevalence of hypodontia in Koreans. Oral Dis. 2008;14:620-5.

21. Ezoddini AF, Sheikhha MH, Ahmadi H. Prevalence of dental developmental anomalies: a radiographic study. Community Dent Health. 2007;24:140-4.

22. Guideline on dental management of pediatric patients receiving chemotherapy, hematopoietic cell transplantation, and/or radiation therapy. Pediatr Dent. 2016;38:334-42.

23. Dirican B, Ozen J, Beyzadeoglu M, Oysul K, Surenkok S, Sipahi C. In vitro evaluation of head and neck radiation shields used to reduce exit dose. Int J Prosthodont. 2006;19: $462-6$. 